\title{
Researches on Six Lattice-Valued Logic
}

\author{
Hua Li \\ Department of Information Engineering, Hangzhou Polytechnic College, Hangzhou, China \\ Email: zjlihua@126.com
}

Received 2 September 2015; accepted 26 October 2015; published 29 October 2015

Copyright (C) 2015 by author and Scientific Research Publishing Inc.

This work is licensed under the Creative Commons Attribution International License (CC BY).

http://creativecommons.org/licenses/by/4.0/

(c) (i) Open Access

\begin{abstract}
Based on the direct product of Boolean algebra and Lukasiewicz algebra, six lattice-valued logic is put forward in this paper. The algebraic structure and properties of the lattice are analyzed profoundly and the tautologies of six-valued logic system $L_{6} P(X)$ are discussed deeply. The researches of this paper can be used in lattice-valued logic systems and can be helpful to automated reasoning systems.
\end{abstract}

\section{Keywords}

Six Lattice-Valued Logic, Lattice Implication Algebra, Filter, Tautology

\section{Introduction}

Lattice-valued logic is an important case of multi-valued logic, and it plays more and more important roles in artificial intelligence and automated reasoning. Six lattice-valued is a kind of common lattice, which can express logic in real world, such as language values, and evaluation values. It can deal with not only comparable information but also non-comparable information. Therefore, theoretical researches and logic and reasoning systems based on six lattice-valued logic are of great significance.

\section{The Structure of Lattice $L_{6}$}

The set of $L=\{O, a, b, c, d, I\}$ is a lattice, and the order relation of $L$ is shown in Figure 1 . The complement operator " "'”and implication operation " $\rightarrow$ " are defined in Table 1 respectively.

$L$ means an lattice implication algebra.

Then set $A=\{O, I\}, B=\{O, m, I\}$. As $A$ is the true set of classical binary logic, the operation rules of the complement operation and the implication operation are the same with the classical two-valued logic systems. $B$ is the true-value set of Lukasiewicz system with three-valued logic, and complement operations and implication operations are defined in Table 2.

Let $L^{*}=A \times B$, the order relations, disjunctive, conjunctive, complement operation and implication operation 


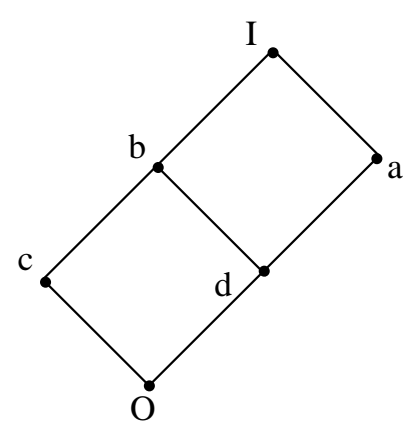

Figure 1. Structure of the six-valued lattice.

Table 1. Computing of the six-valued lattice $L_{6}$.

\begin{tabular}{lllllllll}
\hline$x$ & $x^{\prime}$ & $\rightarrow$ & $O$ & $a$ & $b$ & $c$ & $d$ & $I$ \\
$O$ & $I$ & $O$ & $I$ & $I$ & $I$ & $I$ & $I$ & $I$ \\
$a$ & $c$ & $a$ & $c$ & $I$ & $b$ & $c$ & $b$ & $I$ \\
$b$ & $d$ & $b$ & $d$ & $a$ & $I$ & $b$ & $a$ & $I$ \\
$c$ & $a$ & $c$ & $a$ & $a$ & $I$ & $I$ & $a$ & $I$ \\
$d$ & $b$ & $d$ & $b$ & $I$ & $I$ & $b$ & $I$ & $I$ \\
$I$ & $O$ & $I$ & $O$ & $a$ & $b$ & $c$ & $d$ & $I$ \\
\hline
\end{tabular}

Table 2. Computing of $L_{3}$.

\begin{tabular}{cccccc}
\hline$x$ & $x^{\prime}$ & $\rightarrow$ & $O$ & $m$ & $I$ \\
$O$ & $I$ & $O$ & $I$ & $I$ & $I$ \\
$m$ & $m$ & $m$ & $m$ & $I$ & $I$ \\
$I$ & $O$ & $I$ & $O$ & $m$ & $I$ \\
\hline
\end{tabular}

on $L$ are defined as follows:

For any $(a, b) \in L^{*}, \quad(c, d) \in L^{*}$ :

(1) $(x, y) \leq(z, r)$, if and only if $x \leq z$ and $y \leq r$.

(2) $(x, y)=(z, r)$, if and only if $x=z$ and $y=r$.

(3) Under other circumstances, $(x, y)$ cannot be compared with $(z, r)$.

(4) $(x, y) \wedge(z, r)=(x \wedge z, y \wedge r),(x, y) \vee(z, r)=(x \vee z, y \vee r)$.

(5) $(x, y) \rightarrow(z, r)=(x \rightarrow z, y \rightarrow r)$.

(6) $(x, y)^{\prime}=\left(x^{\prime}, y^{\prime}\right)$.

The $L^{*}$ constitute a six element lattice and its operation diagram is shown in Hasse Figure 2.

Theorem 1. $L$ is isomorphic lattice implication of $L^{*}$.

Proof:

Obviously, we can construct a upward one-to-one mapping from $L$ to $L^{*}: \quad f: L \rightarrow L^{*}$, making

$$
\begin{array}{lll}
f(O)=(O, O), & f(a)=(O, I), & f(b)=(I, m) \\
f(c)=(I, O), & f(d)=(O, m), & f(I)=(I, I)
\end{array}
$$

Clearly $f$ is conjunctive homomorphic mapping and disjunctive homomorphism mapping.

Here is the proof that $f$ is complement homomorphic mapping and implication homomorphism mapping.

According to the definition of implication operations and complement operations, it can be easily obtained in Table 3. 


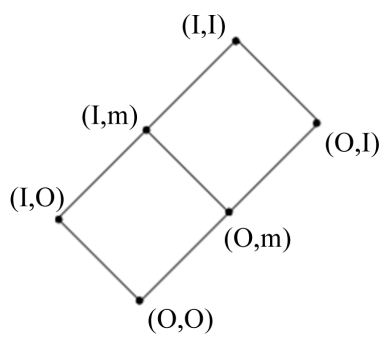

Figure 2. Six-valued lattice generated by the direct product.

Table 3. Six-valued lattice generated by the direct product.

\begin{tabular}{ccccccccc}
\hline$x$ & $x^{\prime}$ & $\rightarrow$ & $(O, O)$ & $(O, I)$ & $(I, m)$ & $(I, O)$ & $(O, m)$ & $(I, I)$ \\
$(O, O)$ & $(I, I)$ & $(O, O)$ & $(I, I)$ & $(I, I)$ & $(I, I)$ & $(I, I)$ & $(I, I)$ & $(I, I)$ \\
$(O, I)$ & $(I, O)$ & $(O, I)$ & $(I, O)$ & $(I, I)$ & $(I, m)$ & $(I, O)$ & $(I, m)$ & $(I, I)$ \\
$(I, m)$ & $(O, m)$ & $(I, m)$ & $(O, m)$ & $(O, I)$ & $(I, I)$ & $(I, m)$ & $(O, I)$ & $(I, I)$ \\
$(I, O)$ & $(O, I)$ & $(I, O)$ & $(O, I)$ & $(O, I)$ & $(I, I)$ & $(I, I)$ & $(O, I)$ & $(I, I)$ \\
$(O, m)$ & $(I, m)$ & $(O, m)$ & $(I, m)$ & $(I, I)$ & $(I, I)$ & $(I, m)$ & $(I, I)$ & $(I, I)$ \\
$(I, I)$ & $(O, O)$ & $(I, I)$ & $(O, O)$ & $(O, I)$ & $(I, m)$ & $(I, O)$ & $(O, m)$ & $(I, I)$ \\
\hline
\end{tabular}

It can be seen from the Table $3, f$ is the implication operations and the complement operations homomorphic.

In summary, we proofed that:

For any $x, y \in L, f\left(x^{\prime}\right)=(f(x))^{\prime}, \quad f(x * y)=f(x) * f(y)$, where $*$ is one of disjunctive, conjunctive, complement operation.

Thus $L$ and $L^{*}$ is isomorphic lattice implication.

\section{The Property and Language of Lattice $L_{6}$}

Due to $L_{6}$ is a lattice implication algebra, it not only has all the properties of lattice implication algebra but also properties as follows.

Theorem 2. As shown the six-valued lattice $L_{6}$ in Figure 1, the implication operation satisfies the following properties: For any $x, y, z \in L_{6}$ :

(1) $z \leq y \rightarrow x$ iff $y \leq z \rightarrow x$.

(2) $z \rightarrow(y \rightarrow x) \geq(z \rightarrow y) \rightarrow(z \rightarrow x)$.

(3) $(x \rightarrow y) \vee\left((x \rightarrow y) \rightarrow\left(x^{\prime} \vee y\right)\right)=I$.

(4) $y \rightarrow z \leq(x \rightarrow y) \rightarrow(x \rightarrow z)$.

(5) $x \rightarrow(y \rightarrow z)=y \rightarrow(x \rightarrow z)$.

(6) $x \rightarrow y \leq(x \vee z) \rightarrow(y \vee z)$.

Theorem 3. As the true subset of $L_{6}, L_{0}=\{O, I, a, c\}$ is a sub lattice implication algebra. What's more, $L_{0}$ is a Boolean algebra, and the implication arithmetic of it meets that: for any $x, y \in L_{0}, x \rightarrow y=x^{\prime} \vee y$.

Proof: It is clearly that $L_{0}$ is a sub lattice of $L_{6}$. For any $x, y \in L_{0}, x^{\prime} \in L_{0}, \quad x \rightarrow y \in L_{0}$, therefore when regarding $L_{6}$, the operation of $L_{0}$ is closed, that is to say, $L_{0}$ is a sub lattice implication algebras of $L_{6}$.

It can be verified easily: for any $x, y \in L_{0}, x \rightarrow y=x^{\prime} \vee y$. Meeting the of Boolean algebra axiom, $L_{0}$ is a Boolean algebra.

Any sub-set of power set lattice in a collection is called the set lattice for the collection. The isomorphism from a lattice $L$ to a set lattice $B(X)$ in collection $X$ is named as a isomorphic representation $L$ by $B(X)$, which can be denoted as $L$ for abbreviation. Through establishing the lattice representation, lattice language can be simplified, which is very important for studying the structure and properties of the lattice.

Definition 1 [1]. Let $L$ is a lattice, an element $x \in L$ is called as an join-irreducible element, if 
(1) $x \neq O$ (when there is a minimum of $O$ when $L$ );

(2) For any $a, b \in L$, if $x=a \vee b$, then $x=a$ or $x=b$.

Assume $L$ is a finite distributive lattice, $\mathfrak{I}(L)$ denotes the set of all join-irreducible element in the collection, and all the join-irreducible element in $L$ can form under set lattice (i.e. ideal Lattice) according to the order relation which can be indicated as $O(\Im(L))$. Then we have the following conclusions:

Theorem 4 [2]. Let $L$ is a finite distributive lattice, and mapping can be constructed as follows:

$$
\begin{aligned}
& \eta: L \rightarrow O(\Im(L)) \\
& \eta(a)=\{x \in \mathfrak{I}(L) \mid x \leq a\}
\end{aligned}
$$

The $\eta$ is the lattice isomorphism from $L$ to $O(\Im(L))$.

Theorem 5 [2]. Let $L$ is a finite distributive lattice, then the following equivalent hold:
1) $L$ is a distributive lattice;
2) $L \cong O(\Im(L))$;
3) $L$ is isomorphic to a set lattice;
4) For any $n \geq 0, L$ is isomorphic to $2^{n}$ sub lattice.

According to Theorem 5 , theorem representation of six lattice-valued $L_{6}$ can be got easily.

Theorem 6. As shown the six-valued lattice $L_{6}$ in Figure 1, conclusions as follows can be got:

(1) The set of join-irreducible element in $L_{6}$ is $\mathfrak{J}\left(L_{6}\right)=\{a, b, c\}$, and its order relation are shown in Figure 3.

(2) The under set lattice (i.e. ideal lattice), which is the set of all the join-irreducible element and forms according to its order relation, is $O\left(\mathfrak{I}\left(L_{6}\right)\right)=\{\phi,\{c\},\{a\},\{b, c\},\{a, c\},\{a, b, c\}\}$.

(3) The Hasse diagram $O\left(\Im\left(L_{6}\right)\right)$ of the ideal lattice of $L_{6}$, which forms through inclusion relation, is shown in Figure 4. Form the figure, we can see that $L_{6}$ is isomorphic of lattice implication to its ideal lattice $O\left(\Im\left(L_{6}\right)\right)$. Lattice implication isomorphism $\eta$ is defined as follows:

$$
\begin{aligned}
& \eta: L \rightarrow O(\Im(L)) \\
& h(O)=\phi, \eta(a)=\{b, c\}, \eta(b)=\{a, c\}, \eta(c)=\{a\} \\
& \eta(d)=\{c\}, \eta(I)=\{a, b, c\}
\end{aligned}
$$

\section{The Filter of Lattice $L_{6}$}

Since all Lukasiewicz algebras are lattice implication algebra [1], it can be proved that Lukasiewicz algebra filters are trivial.
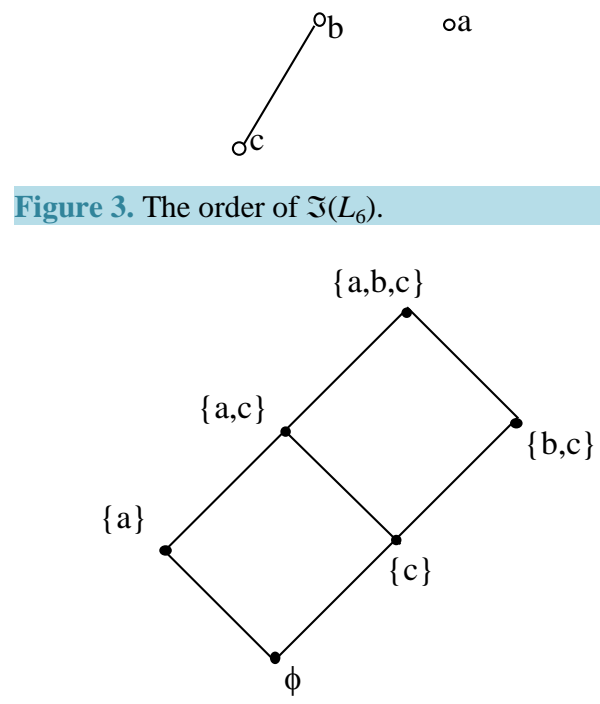

Figure 4. The ideal lattice of $O\left(\Im\left(L_{6}\right)\right)$. 


\section{Theorem 7.}

(1) The finite chain of Lukasiewicz only contains trivial filters.

(2) Lukasiewicz algebra [0,1] only contains trivial filters.

Proof: (1) Let's set $L=L_{n}=\left\{0, \frac{1}{n-1}, \frac{2}{n-1}, \cdots, \frac{n-2}{n-1}, 1\right\}(n=2,3, \cdots)$. Specific operations are as follows:

For any $x, y \in L$,

$$
\begin{aligned}
& x \vee y=\max \{x, y\}, \quad x \wedge y=\min \{x, y\} \\
& x^{\prime}=1-x, \quad x \rightarrow y=\min \{1,1-x+y\}
\end{aligned}
$$

It is clearly that set $\{1\}$ and $L$ are trivial filters in $L$. we can proof that $L$ don't contain any other trivial filters.

From Theorem 6 we can see that filters in $L$ are ideal dual filters of $L$, and the set of ideal dual filters of $L$ are upper set of $L$.

If $F=\left\{\frac{k}{n-1}, \frac{k+1}{n-1}, \cdots, 1\right\}$ (where $k \geq 1$ ) is a filter of $L$, then

$\frac{k}{n-1} \rightarrow \frac{k-1}{n-1}=\min \left\{1-\frac{k}{n-1}+\frac{k-1}{n-1}, 1\right\}=\frac{n-2}{n-1} \in F$,

And $\frac{k}{n-1} \in F$, so it can be seen that the definition of filters: $\frac{k-1}{n-1} \in F$.

This shows that $F=L$, so it demonstrated that $L$ don't contain any other trivial filters.

(2) Let $L=[0,1]$, its upper operation is the same as defined $C_{2}$.

It is clearly that set $\{1\}$ and $L$ are trivial filters in $L$. we can proof that $L$ don't contain any other trivial filters.

We can see that filters in $L$ are ideal dual filters of $L$, and the set of ideal dual filters of $L$ are upper set of $L$. So the filter of $L$ must be an interval containing greatest element 1.

Firstly, we can proof that the filter of $L$ must be a closed interval.

Let us set $F=(u, 1)$ is a filter of $L$, where $0<u<1$, for any $x$, satisfies $u<x<1$, then $x \in F$, and $x \rightarrow u=\min \{1,1-u+x\}=1-u+x>u \in F$, conclusion can get $u \in F$.

This shows that $F$ is a closed interval.

Secondly, assume $F=[u, 1]$ is a filter of $L$, where $0<u<1$.

For any $x$, making $u<x<1$ and $x+u \geq 1$, then

$$
u \rightarrow(x+u-1)=\min \{1-u+(x+u-1), 1\}=x \in F
$$

thereby $x+u-1 \in F$, that is contradictory, because $0 \leq x+u-1<u$.

So $F$ is an interval.

This proves that Lukasiewicz interval only have trivial filters.

As a special case of Theorem 7, we have the following corollary.

Corollary 1. $C_{2}=\{O, I\}$ and $L_{3}=\{O, m, I\}$ only contain trivial filters.

Theorem 8. The six element lattice only contains the following four filters:

$\{I\}, L_{6}, \quad F_{a}=\{I, a\}, \quad F_{b c}=\{I, b, c\}$.

Proof: According to Theorem $1, L_{6}$ can be seen as the direct product of $C_{2}$ and $L_{3}$. According to Corollary 1 , $C_{2}=\{O, I\}$ and $L_{3}=\{O, m, I\}$ only contain trivial filters. As followed:

The filters of $C_{2}=\{O, I\}$ are $\{I\}$ and $\{O, I\}$.

The filters of $L_{3}=\{O, m, I\}$ are $\{I\}$ and $\{O, m, I\}$.

It is easy to know, the filters of $L_{6}$ are the direct products of the filters of $C_{2}$ and the filters of $L_{3}$. So the filters of $L_{6}$ are as followed:

$\{(I, I)\},\{(I, I),(O, I)\},\{(I, I),(I, m),(I, O)\}$ and $L_{6}$ itself.

In other words: The six element lattice $L_{6}$ only contains the following four filters:

$\{I\}, L_{6},\{I, a\},\{I, b, c\}$.

\section{The Tautologies of Lattice-Valued Logic System $L_{6} P(X)$}

Here we take the lattice-valued logic system $L_{6} P(X)$ into consideration, and discuss its tautologies and $F$-tauto- 
logies, the true value domain is $L_{6}$.

It is easy to verify:

$$
L_{6}=C_{2} \times L_{3}
$$

where $C_{2}$ is a Boolean algebra $\{O, I\}, L_{3}$ is a Lukasiewicz algebra $\{O, m, I\}$.

Theorem 9. (The definition of tautologies in $L_{6} P(X)$ [3]) The tautologies in six lattice-valued logic system $L_{6} P(X)$ process the following relationship:

(1) $T^{L_{6}}=T^{C_{2}} \cap T^{L_{3}}=T^{L_{3}}$.

(2) $T_{a}^{L_{6}}=T^{L_{3}}$.

(3) $T_{b}^{L_{6}}=T^{C_{2}} \cap T_{m}^{L_{3}}=T^{C_{2}}$.

(4) $T_{c}^{L_{6}}=T^{C_{2}}$.

(5) $T_{d}^{L_{6}}=T_{m}^{L_{3}}=T^{C_{2}}$.

Proof: It is noticed that the tautologies in Lukasiewicz three-valued logic system process the following relationship:

$$
T^{L_{3}} \subset T_{m}^{L_{3}}=T^{C_{2}}
$$

Proof of this theorem can be obtained.

From Theorem 7, the six element lattice $L_{6}$ only contains four filters as followed:

$\{I\}, L_{6}, \quad F_{a}=\{I, a\}, \quad F_{b c}=\{I, b, c\}$.

Therefore, its non-trivial filters are $F_{a}=\{I, a\}, F_{b c}=\{I, b, c\}$.

We can get the definition of $F$-tautologies in six lattice-valued logic system $L_{6} P(X)$ as Theorem 8 similarly.

Theorem 10. (The definition of $F$-tautologies in $L_{6} P(X)$ [4]) The $F$-tautologies in six lattice-valued logic system $L_{6} P(X)$ process the following relationship:

(1) $T_{F_{a}}^{L_{6}}=T^{L_{3}}$.

(2) $T_{F_{b c}}^{L_{6}}=T^{C_{2}}$.

Proof:

Since $T(f)(A) \neq T(g)(A)$, so $T$ is an injection.

Clearly $T$ is a surjection. For any $\left(U_{1}, \Psi_{L}\left(U_{1}\right)\right),\left(U_{2}, \Psi_{L}\left(U_{2}\right)\right) \in|\aleph(L)|$,

$\forall \hat{\mathfrak{R}}_{f} \in H_{S \aleph(L)}\left(\left(U_{1}, \Psi_{L}\left(U_{1}\right)\right),\left(U_{2}, \Psi_{L}\left(U_{2}\right)\right)\right), G$ has the inverse image.

Thus $G$ is an isomorphic functor of $\aleph(L)$.

As isomorphic relationship means an equivalence relation, so $S \aleph(L)$ and $\mathfrak{I}(L)$ are isomorphic.

\section{Conclusion}

In this paper, the six element lattice is built by the direct product of Boolean algebra and Lukasiewicz algebra; the operation of the lattice is defined; the structures, properties and filters are studied; finally the tautologies and $F$-tautologies of the six lattice-valued logic system are discussed. The results of this paper can be applied to lattice-valued logic systems and automated reasoning applications.

\section{Acknowledgements}

The work is supported by the project of Zhejiang province education department of China, Grant No. Y201326675.

\section{References}

[1] Yang, X. and Yun, Q.K. (1995) Fuzzy Lattice Implication Algebra. Southwest Jiaotong University, 2, 121-127.

[2] Xu, Y., Ruan, D. and Liu, J. (2004) Progress and Prospect in Lattice-Valued Logic Systems Based on Lattice Implication Algebras. Proceedings of the 6th International FLINS Conference Applied Computational Intelligence, 29-34. 
http://dx.doi.org/10.1142/9789812702661_0009

[3] Min, H.T. (1996) Georgia, Sequencing Primer Theory and Its Application. Southwest Jiaotong University Press.

[4] Fang, S. and Mei, Z.F. (2010) Six Yuan-Based Language of Logic Attributed True Value Method. Guangxi Normal University, 3, 118-122. 